\title{
Sensitization to Cor a 9 and Cor a 14 is highly specific for a severe hazelnut allergy in Dutch children and adults
}

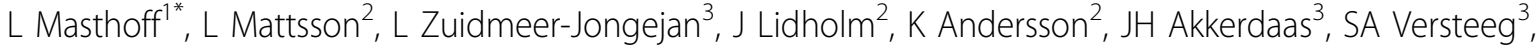 \\ C Garino ${ }^{4}$, Y Meijer ${ }^{5}$, P Kentie ${ }^{5}$, A Versluis ${ }^{1}$, CF den Hartog Jager ${ }^{1}$, CA Bruijnzeel-Koomen ${ }^{1}$, AC Knulst ${ }^{1}$, R van Ree ${ }^{3}$, \\ E van Hoffen ${ }^{1}$, SG Pasmans ${ }^{1}$
}

From Food Allergy and Anaphylaxis Meeting (FAAM 2013)

Nice, France. 7-9 February 2013

\section{Background}

Component-resolved diagnosis has been shown to improve diagnosis of food allergy. The aim of this study was to evaluate whether component-resolved diagnosis may help to identify patients at risk of severe allergic reactions to hazelnut.

\section{Methods}

A total of 161 hazelnut-sensitized patients were included: 40 children and 15 adults with objective symptoms in DBPCFC and 24 adults with a convincing severe history were compared to 41 children and 41 adults with no or subjective symptoms in DBPCFC (grouped together). IgE levels to hazelnut extract and single components were analyzed with ImmunoCAP.

\section{Results}

IgE to hazelnut extract was significantly higher in children with severe than with no or mild symptoms. Sensitization to rCor a 1.04 was common among both children and adults, while sensitization to rCor a 8 was rare. In $13 \%$ of children and $49 \%$ of adults with a severe hazelnut allergy, only sensitization to rCor a 1.04 was observed and not to other water-soluble allergens. Sensitization to nCor a 9 and/or rCor a 14 was strongly associated with a severe hazelnut allergy. Using adapted cutoff levels, a diagnostic discrimination between severity groups was obtained. IgE to either nCor a $9 \geq 1 \mathrm{kU}_{\mathrm{A}} / \mathrm{L}$ or rCor a $14 \geq 5 \mathrm{kU}_{\mathrm{A}} / \mathrm{L}$ (children) and IgE to either
nCor a $9 \geq 1 \mathrm{kU}_{\mathrm{A}} / \mathrm{L}$ or rCor a $14 \geq 1 \mathrm{kU}_{\mathrm{A}} / \mathrm{L}$ (adults) had a specificity of $>90 \%$ and accounted for $83 \%$ of children and $44 \%$ of adults with a severe hazelnut allergy.

\section{Conclusion}

Sensitization to Cor a 9 and Cor a 14 is highly specific for patients with objective symptoms in DBPCFC as marker for a more severe hazelnut allergic phenotype.

\section{Disclosure of interest}

None declared.

\section{Author details}

${ }^{1}$ Dermatology/Allergology, University Medical Center Utrecht, Utrecht, the Netherlands. ${ }^{2}$ Thermo Fisher Scientific, Uppsala, Sweden. ${ }^{3}$ Experimental Immunology, Academic Medical Center, Amsterdam, the Netherlands. ${ }^{4}$ Pharmaceutical Sciences, Drug \& Food Biotechnology Center, University of Piemonte Orientale "A. Avogadro", Novara, Italy. ${ }^{5}$ Pediatric Pulmonology/ Allergology, University Medical Center Utrecht, Utrecht, the Netherlands.

Published: 25 July 2013

doi:10.1186/2045-7022-3-S3-014

Cite this article as: Masthoff et al: Sensitization to Cor a 9 and Cor a 14 is highly specific for a severe hazelnut allergy in Dutch children and adults. Clinical and Translational Allergy 2013 3(Suppl 3):014. 